\title{
INTRODUCTORY NOTES FOR THE ACTA IMEKO TC3, TC5, TC16, AND TC22 SPECIAL ISSUE 2020
}

\author{
Andy Knott ${ }^{1}$, Dirk Röske ${ }^{2}$, Renato Reis Machado ${ }^{3}$, Jay Hendricks ${ }^{4}$, Dominik Pražák ${ }^{5}$, Davor Zvizdić 6 , \\ Lovorka Grgec Bermanec ${ }^{6}$, Gustavo Ripper ${ }^{3}$ \\ ${ }^{1}$ National Physical Laboratory (NPL), United Kingdom \\ ${ }^{2}$ Physikalisch-Technische Bundesanstalt (PTB), Germany \\ ${ }^{3}$ National Institute of Metrology, Quality and Technology (INMETRO), Brazil \\ ${ }^{4}$ National Institute of Standards and Technology (NIST), USA \\ ${ }^{5}$ Czech Metrology Institute (CMI), Czech Republic \\ ${ }^{6}$ Faculty of Mechanical Engineering and Naval Architecture, University of Zagreb, Croatia
}

Distinguished Readers,

The joint IMEKO TC3, TC5, TC16, and TC22 International Conference was first scheduled to take place in Cavtat-Dubrovnik, Croatia, in May 2020. When the Coronavirus appeared earlier this year, the organisers decided to postpone the conference to November 2020, hoping that the occurrence of infections could be reduced to a minimum by autumn. Due to the continuing spread of the virus which turned into a pandemic, the conference was again postponed, this time to 2022, as the XXIII IMEKO World Congress was already scheduled for the year 2021. However, more than 100 extended abstracts had already been submitted to the conference and peer-reviewed, with about 80 scientific papers ready for publication by November 2020 . The Chairs of the International Programme Committee therefore decided to offer the authors the possibility to publish these papers in a Special Issue of Acta IMEKO and nearly all authors have agreed.

Technical Committee 3 of IMEKO (IMEKO TC3 - "Measurement of Force, Mass and Torque") was established as a Technical Committee for the "Measurement of Force and Mass" in 1967. Considering the growing importance and new developments in the field of torque measurement, the name of the TC changed to "Measurement of Force, Mass and Torque" in 1998. The main topics include: mass, force, torque, and dynamic measurement of these quantities.

Technical Committee 5 of IMEKO (IMEKO TC5 - "Hardness Measurement") was established in 1973. TC5 provides a forum for the dissemination of measuring techniques and principles, and discussion about problems in connection with these techniques and principles, related to the physical quantities of hardness: Rockwell, Brinell, Vickers, instrumented indentation and Leeb hardness scales, as well as new definitions for hardness scales.

Technical Committee 16 of IMEKO (IMEKO TC16 - "Pressure and Vacuum Measurement") was established in 1986. Its main topics include: quantum realisations of the pascal, pressure standards, vacuum standards, mass spectrometry and primary leaks, pressure and vacuum sensors, dynamic pressure measurements, and recent, novel, and new advances in pressure and vacuum technology.

Technical Committee 22 of IMEKO (IMEKO TC22 - "Vibration Measurement") was founded in 2005. Its main topics include: vibration sensors, vibration measuring devices, single and multi-axial vibration, dynamics of interacting systems, and dynamics of acceleration-related quantities.

IMEKO technical committees TC3, TC5, TC16, and TC22 have a long history of joint events in many combinations and forms. This is to be expected because the topics of the four TCs are interrelated and often depend on each other's achievable uncertainties and developments. Those events and related publications have always had a significant impact on international interchange of scientific and technical information and the enhancement of international co-operation among scientists and engineers from research and industry in the IMEKO TC3, TC5, TC16, and TC22 fields of activity. 
The papers in this issue appear in the order given by the TC numbers with two general papers at the end. The layout of the papers differs slightly from the usual layout of articles in regular issues of Acta IMEKO. This reflects the fact that the papers had been submitted to a conference using the "conference layout". We have just replaced the header by that of the journal on the first page of each article and the footer on each page. We also needed to adjust the page margins to keep the structure of the papers. We are convinced that these format changes have no influence on the scientific content of the articles. And here is what to expect on the next 415 pages:

\section{Papers related to IMEKO TC3 - Measurement of Force, Mass and Torque}

There are eleven papers in the general area of mass standards - the first nine cover a wide range of subjects related to mass laboratory activities including cleaning, simultaneous mass and volume determination, weighing of non-metal weights, weight handling, air buoyancy effects, and buoyancy artefact development (pp. 4-46), while the final two relate to current Kibble balance projects (pp. 47-57). These are followed by four papers related to weighing applications - one analyses the structure of a truck scale (pp. 58-62), two look at weighing-in-motion systems (pp.63-74), and the fourth covers vibration compensation in dynamic measurements (pp. 75-78). The one paper in the density area looks at a comparison of viscoelastic samples (pp. 79-84). Within the force standards topic, there are six papers related to the design, analysis, calibration of, and comparison between force standard machines (pp. 85-108), two papers on microforce measurement (pp. 109-117), and a further two papers on dynamic force (pp. 118-128). In the area of force applications, there are five papers that look at different aspects of the performance of force measurement systems (pp. 129-155), followed by three papers that consider more industrial applications, including bolt stretcher calibration, alignment tests, and thread-grinding force measurement (pp. 156-167). The six torque papers comprise two looking at torque standard machines, two dealing with calibration procedures and comparison results, and the final two evaluating torque wrenches and transducer sensitivity (pp. 168-199). The TC3 contribution is completed by three papers that look at the instrumentation used for force transducers, in both the static and dynamic regimes (pp. 200-215).

\section{Papers related to IMEKO TC5 - Hardness Measurement}

The papers submitted to TC5 include contributions related to indenter geometries (pp. 216-225), standard machines (pp. 226-249), and comparisons (pp. 250-260). In addition, there are other contributions concerning microscope image resolution (pp. 261-264), the instrumented indentation test unloading curve (pp. 265-269), traceability of small force for Vickers calibration (pp. 270-273), and an unmanned precise measurement device for Rockwell hardness measurements (pp. 274-280).

\section{Papers related to IMEKO TC16 - Pressure and Vacuum Measurement}

TC16 is pleased to present papers on the current challenges and recent advances in pressure and vacuum metrology. First, advances in the world-wide effort to establish a quantum-based realisation of the pascal through refractive index are presented in five papers (pp. 281-309). Under the new SI, adopted in May 2019, the quantum-based pascal has benefited from redefinitions of the kelvin, now traceable to the Boltzmann constant, and the kilogram, now traceable to the Planck constant. Because refractometry depends only on the number-density of the gas and the gas species, it is realised as a primary standard when the refractive index of the gas is derived from quantum chemistry calculations and pressure-induced effects of laser cavity distortions are also independently known. Challenges in putting the quantum pascal into practice abound and these papers address the latest efforts to do so. The second theme of the collection is advances in addressing the challenging need for primary dynamic pressure standards. Two papers on dynamic pressure cover both the issue of developing primary dynamic pressures, and the development of dynamic pressure sensors (pp. 310-318). Finally, while the first two topics address innovation in pressure and vacuum metrology, the third and final theme deals with recent advances in long-established, tried and trusted methods. It would be a mistake to assume that the traditional methods of classical pressure balances, liquid-column manometry, and static expansion techniques have no room for improvement. This final section, eight papers in all, addresses recent 
developments and advances in these important classical techniques (pp. 319-356). TC16 is confident that you will enjoy the entire collection of papers covering these three major and important areas of: 1) the quantum pascal, 2) dynamic pressure, and 3) classical pressure and vacuum metrology.

\section{Papers related to IMEKO TC22 - Vibration Measurement}

The papers submitted to IMEKO TC22 include two contributions related to laser vibrometer calibration using optical methods (pp. 357-364) and four related to low-frequency vibration measurements (pp. 365-382). In addition, there are single contributions covering primary shock calibration using a fast linear drive (pp. 383387), simultaneous multi-axis vibration calibration (pp. 388-393), calibration of digital angular rate sensors (pp. 394-400), and interlaboratory vibration comparison results (pp. 401-406).

\section{General papers}

There are two papers in this category. The first one provides a methodology for estimating the dependence of strain gauge force and torque transducers on the ambient temperature (pp. 407-413). This effect is usually investigated in special chambers in which the transducer is exposed to various temperatures within a given range while the machine is operated under unchanged laboratory conditions. The application of standard uncertainty calculation methods to the linear regression of measurement results using the least-squares approach was investigated. The regression analysis of the results allows the temperature coefficient to be determined, including an uncertainty analysis. The second paper deals with accuracy as a concept that is widely used in metrology (pp. 414-418). Difficulties regarding present definitions are explained, present-day use of the concept is explained, the question of whether accuracy is a qualitative concept is discussed, and finally pre-conceptual ideas and a proposal for a new wording of a sustainable definition for accuracy are presented.

\section{Enjoy reading!}

